\title{
Polyrigid and Polyaffine Transformations: A New Class of Diffeomorphisms for Locally Rigid or Affine Registration
}

\author{
Vincent Arsigny, Xavier Pennec, and Nicholas Ayache \\ INRIA Sophia - Projet Epidaure, BP 93, 06902 Sophia Antipolis Cedex, France \\ \{Vincent.Arsigny, Xavier.Pennec, Nicholas.Ayache\}@Sophia.Inria.fr
}

\begin{abstract}
We introduce a novel family of geometrical transformations for the non-rigid registration of medical images, called polyrigid and polyaffine. These transformations have nice mathematical properties, including differentiability and invertibility (i.e diffeomorphism). They are parameterized by a small number of intuitive parameters which allow to recover locally rigid and locally affine deformations often encountered in medical imagery. These transformations are a nice alternative to classical B-Spline transformations (which do not guaranty invertibility). Preliminary experiments on synthetic and real images already illustrate the potentialities and good properties of this approach.
\end{abstract}

\section{Introduction}

The registration of medical images is in general a difficult problem, and numerous methods and tools have been already devised to address this task 11. In this paper, we do not focus on the similarity metric, but rather on the geometrical aspects of the registration process, and more precisely on the family of transformations used to establish dense correspondences between anatomical images.

At the beginning of the spectrum, we have simple parametric transformations such as rigid or affine transformations, which have a very small number of degrees of freedom, and can be efficiently used for intra-patient registration. Other types of transformations, such as those parameterized via B-Splines [2], Thin-Plate-Splines 3, finite elements mechanical models 4] or more general deformable models can have an arbitrary number of degrees of freedom and be used for both inter-subject or intra-subject registration. At the end of the spectrum, deformation fields defining a displacement at every voxel exhibit the highest number of degrees of freedom [5677, and can be used for inter-subject registration.

Each of the above transformations has its particular domain of application. However, in the case of anatomical structures incorporating rigid elements (such as bone articulations, or structures which are subject to simple local deformations, like histological slices), we believe that none of them is fully appropriate. Rigid and affine transformations clearly don't have enough degrees of freedom. On the contrary, deformation fields have too many and thus can be easily misled 
by local minima of the similarity criterion. For the existing intermediate transformations, e.g. B-Splines [2], the degrees of freedom of the transformation are not really adapted as many control points are required to reconstruct several locally rigid behaviors, especially when rotations are substantial.

Our goal in this paper is to define new parametric transformations that exhibit a locally rigid or affine behavior, and that can be efficiently implemented. Also, a very desirable property is invertibility, which is not guaranteed in the approaches based on splines or other interpolation techniques, except in the case of Geodesic Interpolating Splines [8].

An approach was proposed in 9 to smoothly interpolate a deformation outside any finite set of independent rigidly moving regions. Since these regions are arbitrary in shape, it is not straightforward to use this model for inference (i.e. non-rigid registration). Moreover, the invertibility of the interpolated transformation is not always ensured.

Our idea is to use simple fuzzy regions defined by very few parameters: mainly the position of the center, a typical radius of influence and the associated rigid or affine transformation. We show in Sec. 2 that a simple average of the displacement induced by each region leads to invertibility problems. Thus, we develop an infinitesimal approach where the displacement is obtained by the integration of the average speed. To address the implementation efficiency, we investigate several numerical schemes. The result is a new family of invertible and fully parametric transformations that we called polyrigid and polyaffine transforms. We show in Sec. 3 that polyrigid transformations are well-suited for the nonrigid registration of articulated-like object. This is exemplified on 2D histological slices.

\section{Theoretical Framework}

The polyrigid/affine transformations are related to different topics: the interpolation of irregularly spaced data, as addressed by Sheppard in [10] and revisited in [9] and the theory of ordinary differential equations (ODE), which provides tools for creating diffeomorphic transformations 811 .

\subsection{Point-Based Interpolation and Parametric Transformations}

To model transformations $T(x)$ having several distinct rigid or affine behaviors, we propose to use anchor points, denoted $\left(a_{i}\right)_{i \in 1 \cdots N} \in\left(\mathbb{R}^{n}\right)^{N}$. These points represent the core of the influence of each local transformation $T_{i}: \mathbb{R}^{n} \rightarrow \mathbb{R}^{n}$, such that whenever $x$ is "close" in some sense to $a_{i}$, we should have $T(x) \approx T_{i}(x)$. Choosing points (rather than regions) to anchor transformations is a convenient choice to explicit the transformation parameters, thus facilitating their inference. A simple way to mix the influence of each transformation is to average their induced displacement:

$$
T(x)=\frac{\sum_{i} w_{i}(x) T_{i}(x)}{\sum_{i} w_{i}(x)}
$$


with some positive "weights" function $w_{i}(x)$ describing the influence of each component. In the current implementation, we use Gaussians $w_{i}(x)=p_{i} \cdot G_{\left(a_{i}, \sigma_{i}\right)}(x)$, where the standard deviations $\sigma_{i}$ control the rate of decay and the $p_{i}$ 's rank the global influence of the each component. This way, the obtained transformation is smooth, but there is no easy way to express its inverse, if it ever exists.

\subsection{Diffeomorphic Transformations via ODEs}

In order to address the invertibility problem, we chose an infinitesimal approach, in which averaging is compatible with inversion. The idea is to average the infinitesimal displacements (instantaneous speed) generated by each component, and obtain the total displacement by integration the resulting flow. To see this, let us recall that a point moving under the action of a rigid transformation $T_{i}(x)=R_{i} x+t_{i}\left(R_{i}\right.$ is the rotation matrix and $t_{i}$ the translation part) can be viewed as a point following a trajectory governed by the following ODE:

$$
\dot{x}(s)=V_{i}(x, s)=t_{i}+A_{i}\left(x-s t_{i}\right) \text { for time } s \in[0,1] .
$$

This ODE simply comes from the derivation w.r.t. $s$ of the trajectory equation $x(s)=s t_{i}+e^{s A_{i}} \cdot x(0) . A_{i}$ is the smallest skew symmetric matrix associated with the rotation $e^{A_{i}}=R_{i}$ (cf. [12] for a determination of $A_{i}$ ). If we denote $\Phi_{i}(x, s)$ the flow associated with this ODE, we have $T_{i}()=.\Phi_{i}(., 1)$, which means that $T_{i}(x)$ is obtained as the position at time $s=1$ of a mobile starting at time $s=0$ at $x$ and moving according to Eq. (1). In order to obtain a transformation $T$ that encompasses all the influences of a system of transformations $\left(T_{i}\right)_{i}$, we average the speed vectors $V_{i}(x, s)$ with the weights $w_{i}(x)$ and let any given point evolve between time 0 and 1 to obtain its new position. The new ODE is written:

$$
\dot{x}(s)=V(x, s)=\frac{\sum_{i} w_{i}(x) V_{i}(x, s)}{\sum_{i} w_{i}(x)} .
$$

Mathematically, equation (2) is nice, for the following two reasons: firstly, $V(x, s)$ is $\mathcal{C}^{\infty}$ in both space and time since all quantities are $\mathcal{C}^{\infty}$ and the denominator never vanishes. Secondly, we may bound the speed at each time $s$ by a radius that depends on the position and the time: there exists three positive constants $C_{1}, C_{2}$ and $C_{3}$ such that $\|V(x, s)\|_{2} \leq C_{1}+C_{2}|s|+C_{3}\|x\|$. This implies that the displacement obtained by integrating the speed is bounded by an exponentially growing radius, but always remains finite:

$$
\forall s,\|\Phi(x, s)\| \leq e^{C_{3}|s|}\left(\|\Phi(x, 0)\|+\left(1-e^{-C_{3}|s|}\right)\left(C_{1} / C_{3}+|s| C_{2} / C_{3}\right)\right) .
$$

The trajectories are therefore defined for all time and the transformation $T=$ $\Phi(., 1)$ is well-defined. This fact combined with the smoothness property ensures (from the classical ODE theory [13]) that $T$ is a diffeomorphism.

As we can see in Fig. (1), polyrigid transformations produce smooth and intuitive trajectories for points, and are clearly invertible, which is not the case 
Direct displacement averaging

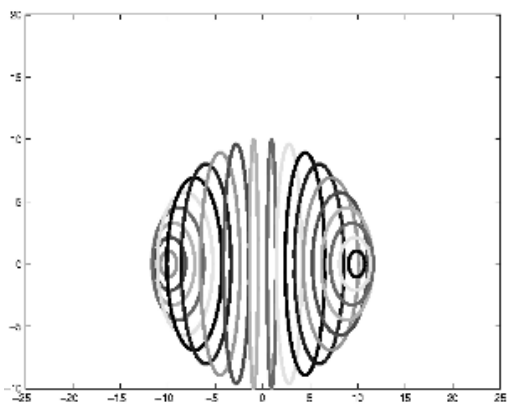

Infinitesimal displacement averaging

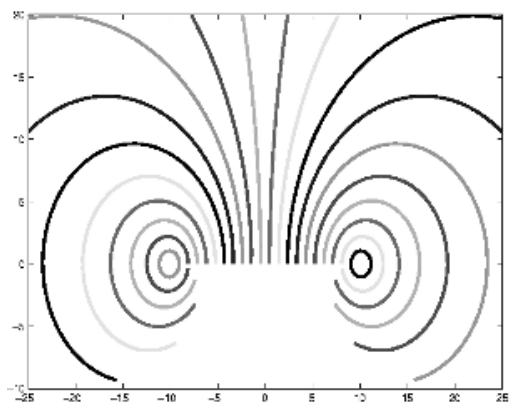

Fig. 1. Comparison between direct and infinitesimal averaging for two $2 \mathrm{D}$ rotations of opposite angle. We displayed the trajectories of a set of points between the two anchor points (rotation centers) for an angle of rotation varying from 0 to $2 \pi$. Near the centers, the transformation is close to rotations and gives circles in both cases. As we go away from the centers, the direct averaging gives ellipsoidal trajectories, whereas the infinitesimal averaging evolves smoothly and remains a diffeomorphism.

when direct averaging is used, mostly when distortions are large. Another advantage is that the inverse is simply obtained by inverting rotation, translation and time parameters.

Like in most Lie Groups, many invertible real matrices $M_{i}$ with $\operatorname{det}\left(M_{i}\right)>0$ are not equal to the exponential of a real matrix. But we have that any element of a real connected Lie Groups is equal to the product of two exponentials [14]. Indeed, the singular value decomposition yields that $M_{i}=e^{A_{i}} \cdot e^{S_{i}}$ with $A_{i}$ and $S_{i}$ respectively skew and symmetric matrices. The equivalent of Eq. (1) for polyaffine transformations is thus:

$$
\dot{x}(s)=t_{i}+\left(A_{i}+e^{s A_{i}} S_{i} e^{-s A_{i}}\right) \cdot\left(x-s t_{i}\right) .
$$

Since all results mentioned before apply also the polyaffine transformations, we thus have exhibited a novel parametric family of smooth and invertible transformations, whose parameters are the same as the usual rigid and affine transformations, plus points to anchor them in space and two additional parameters (the scale $\sigma_{i}$ and the global influence $p_{i}$ ) that control the way their respective influences vary in space.

\subsection{Numerical Implementation}

Practically speaking, Eqs. (2) and (3) have to be solved numerically, since there is no explicit formula except of course in the case of a single component. In order to speed-up the computations, we restricted ourselves to simple numerical schemes (simpler than Runge-Kutta's). The first one is the first-order consistent scheme: sampling the trajectory using $N-1$ intermediate time-points, we may approximate the transformation at time-step $\mathrm{k}$ by applying recursively $k$ times the transformation at time-step 1: 


$$
\left\{\begin{array}{l}
T_{1}^{1 / N}(x, s)=x+\frac{1}{N} V(x, s) . \\
T_{1}^{k / N}(x)=\underbrace{T_{1}^{1 / N}\left(\cdot, \frac{k-1}{N}\right) \circ \cdots \circ T_{1}^{1 / N}}_{k \text { compositions }}(x, 0) .
\end{array}\right.
$$

The complete transformation $T(x)$ is of course approximated by $T_{1}^{N / N}(x)$.

This scheme can be slightly modified to be more efficient: averaging the speed vectors at a given time amounts to assume that they are constant during a time $1 / N$, which is even not exact for a single component. A more efficient strategy is to average the displacements that would be observed if each component was acting alone. Doing so, we obtain a second-order scheme, which is not the optimal one but which is more precise than the first scheme and exact for a single component. For polyrigid transformations, this is simply done by replacing in Eq. (41) $\frac{1}{N} V(x, s)$ with $\frac{\sum_{i} w_{i}(x)\left(\frac{1}{N} t_{i}+\left(e^{\frac{A_{i}}{N}}-I d\right)\left(x-s t_{i}\right)\right)}{\sum_{i} w_{i}(x)}$.

\subsection{Optimization of the Transformations}

To apply our new transformation to inference (i.e. non-rigid registration), it is often necessary to differentiate the transformation $T_{p}(x)$ w.r.t its parameters, denoted by $p$. From the continuous point of view, we have:

$$
\frac{\partial T_{p}}{\partial p}(x)=\frac{\partial \Phi_{p}(x, 1)}{\partial p}=\int_{0}^{1} \frac{\partial V_{p}}{\partial p}\left(\Phi_{p}(x, s), s\right)+\frac{\partial V_{p}}{\partial x}\left(\Phi_{p}(x, s), s\right) d s . \frac{\partial \Phi_{p}(x, s)}{\partial p} .
$$

This formula cannot be used directly, because of the recursive reference to $\frac{\partial \Phi_{p}}{\partial p}$. We must therefore resort to the derivative of the discrete integration scheme:

$$
\frac{\partial T_{p}^{\frac{k}{N}}(x)}{\partial p}=\frac{\partial T_{p}^{\frac{1}{N}}\left(\cdot, \frac{k-1}{N}\right)}{\partial p}\left(T_{p}^{\frac{k-1}{N}}(x)\right)+\frac{\partial T_{p}^{\frac{1}{N}}\left(\cdot, \frac{k-1}{N}\right)}{\partial x}\left(T_{p}^{\frac{k-1}{N}}(x)\right) \cdot \frac{\partial T_{p}^{\frac{k-1}{N}}(x)}{\partial p} .
$$

By recursively applying the derivation process, we obtain a good approximation of the derivative. Thus, one may optimize the transformation in a registration process based on any similarity criterion.

\section{Experiments and Discussion}

Polyrigid transformations are implemented as a new Transform class of ITK [15]. Although the whole implementation is done in 3D, we present in this section preliminary results on $2 \mathrm{D}$ data in order to better display the main features of interest of the polyrigid transformations.

\subsection{Synthetic Examples}

Fig. (2) illustrates the influence of the number of sampling points on the trajectory. One can clearly see that only one time-step (which corresponds to the direct 
averaging of displacements) creates some highly non-linear distortions (almost a discontinuity). With two time-steps, the transformation is already much more regular, and there is almost no evolution after 3 time-steps, which indicates that our numerical scheme is very stable. Notice that this important deformation is only modeled by 2 sites (14 parameters): that would only represent 7 control points for a standard spline interpolation, without the guaranty of invertibility.
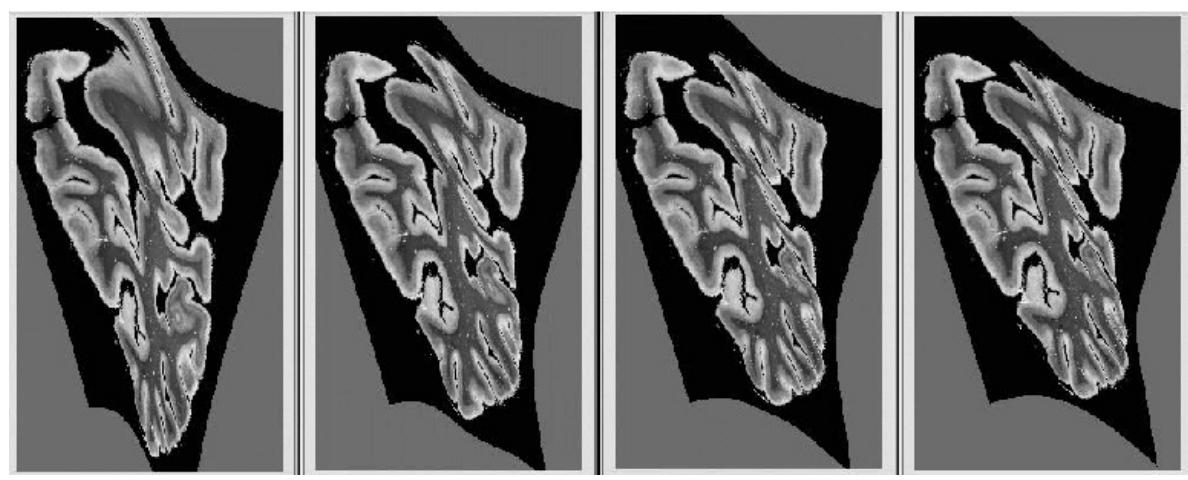

Fig. 2. Influence of the number of sampling points used to compute the transformation: from left to right $\mathrm{N}=1,2,3$, and 20 . One can see that the integration scheme has almost converged with only 3 time-steps.

\subsection{Registration of Histological Slices}

Even if the optimization method still needs to be improved a lot (see [16] for advanced results and for a discussion on initialization and the number of anchor points), we present here preliminary results on the registration of two consecutive histological slices. We believe that our polyrigid transformations are very well adapted to this kind of data as the physical material consists in very thin slices of brain matter with many elongated parts that can rotate around their isthmus during the cutting and fixation process before imaging [17]. An important point in such a registration is to keep very high locally rigid constraints in order to preserve real anatomical differences. Thus, the sought deformation should be close to rigid in large regions, which is exactly what polyrigid transformations provide.

Fig. (3) displays the results of the registration using an affine versus a polyrigid transformation with only 3 rigid components. The non-linear registration algorithm uses a first-order gradient descent: the regular step gradient descent provided by ITK, on the sum of square image difference (SDD) metric. The transformation was initially set to the identity, and the anchors set manually. The main bending is retrieved with only 3 polyrigid components, thus allowing a very fine match of most of the edges, whereas the affine transformation is essentially retrieving a global translation that leaves a large part of the edges 


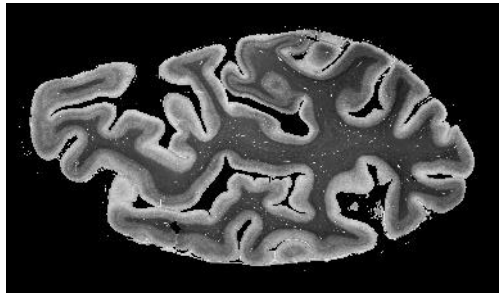

Image $I_{1}$

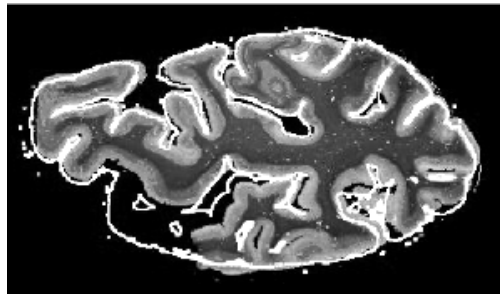

Affine transformation of $I_{2}$

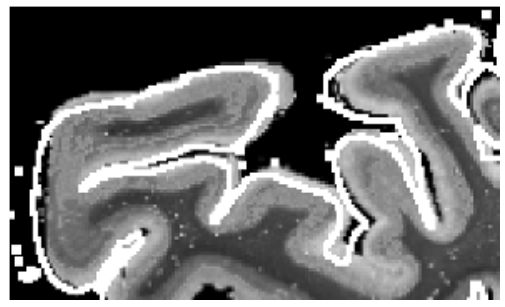

Affine $\left(I_{2}\right)$ : zoom on top left

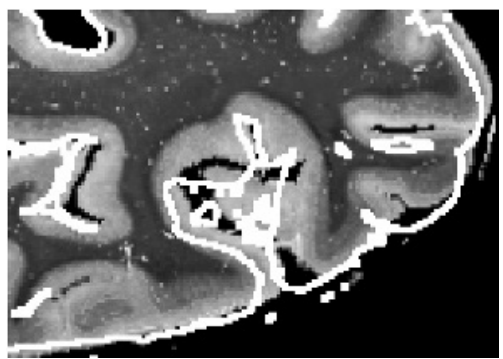

Affine $\left(I_{2}\right)$ : zoom on bottom right

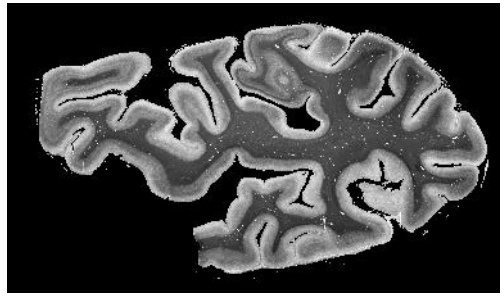

Image $I_{2}$

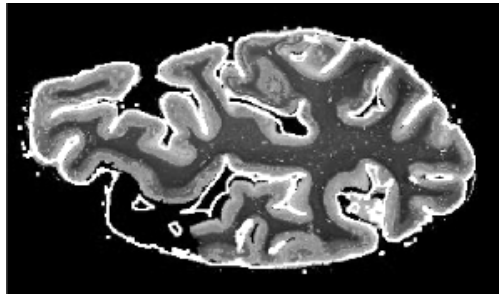

Polyrigid transformation of $I_{2}$

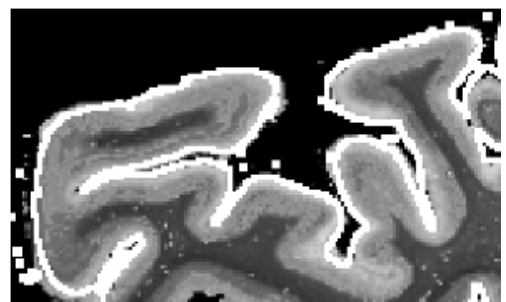

PolyRigid $\left(I_{2}\right)$ : zoom on top left

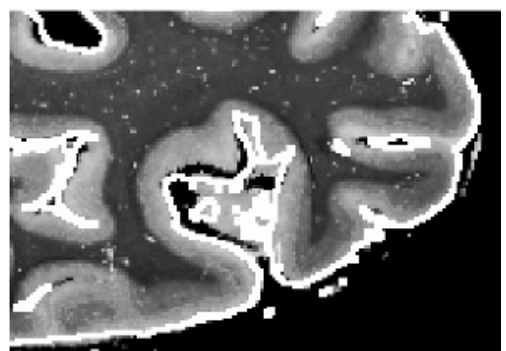

$\operatorname{Polyrigid}\left(I_{2}\right)$ : zoom on bottom right

Fig. 3. Registration of successive histological slices (on the top row) using affine vs polyrigid transformations (on the second row). To assess the quality of the registration, we superimposed on the resampled image $I_{2}$ the contours of image $I_{1}$ (white). On can see on the zooms of the top left part (on the third row) and the bottom right part (on the last row) that the main bending is retrieved with polyrigid, thus allowing to match very accurately most of the edges, which is not the case for the affine registration. A more adapted optimization method is necessary to recover the full bending (see [16]). 
unmatched. The total number of parameters optimized in our polyrigid transformation is 21 (vs 5 for affine), which remains very small compared to most free-form deformation algorithms. With our simple optimization strategy we are only able here to recover a part of the large bending motion of the lower broken girus, but with a more appropriate optimization, this bending can be fully recovered [16. We are currently working on 3D registration experiments, as well as adapting the similarity measure and introducing more complex anatomical knowledge on the region weights in order to adapt the regions influences to the background parts of the images.

Acknowledgments. We are very grateful to P. Thompson, A. Toga, J. Annese and A. Pitiot for providing us with the histological slices through an associated teams collaboration between Epidaure at INRIA and LONI at UCLA.

\section{References}

1. J.B.A. Maintz and M.A Viergever. A survey of medical registration. Medical image analysis, 2(1):1-36, 1998.

2. D. Rueckert, L. I. Sonoda, C. Hayes, D. L. G. Hill, M. O. Leach, and D. J. Hawkes. Non-rigid registration using free-form deformations: Application to breast MR images. IEEE Trans. Medecal Imaging, 18(8):712-721, 1999.

3. F. L. Bookstein. Linear methods for nonlinear maps: Procrustes fits, thin-plate splines, and the biometric analysis of shape variability. In A. Toga, editor, Brain Warping, pages 157-181. Academic Press, 1999.

4. M. Ferrant, S.K. Warfield, C.R.G. Guttmann, R.V. Mulkern, F.A. Jolesz, and R. Kikinis. 3D image matching using a finite element based elastic deformation model. In Proc. of MICCAI'99, LNCS 1679, pages 202-209, 1999.

5. J.-P. Thirion. Image matching as a diffusion process: an analogy with Maxwell's demons. Medical Image Analysis, 2(3):243-260, 1998.

6. Pascal Cachier. Recalage non-rigide d'images médicales volumiques, contributions aux approches iconiques et géométriques. PhD thesis, École Centrale Paris, 2002.

7. C. Chefd'hotel, G Hermosillo, and O. Faugeras. Flows of diffeomorphisms for multimodal image registration. In Proc. of IEEE Int. Symp. on Biomedical Ima., Washington D.C, july 8-11 2002.

8. Vincent Camion and Laurent Younes. Geodesic interpolating splines. In M. Figuiredo, J. Zerubia, and A.K. Jain, editors, Proc. of Energy Minimization Methods in Comp. Vis. and Pat. Rec. (EMMCVPR;01), LNCS 2134, pages 513-527, 2001.

9. J.A. Little, D.L.G. Hill, and D.J. Hawkes. Deformations incorpotationg rigid structures. Computer Vision and Image Understanding, 66(2):223-232, May 1996.

10. D. Sheppard. A two-dimensionnal interpolation function for irregularly spaced data. In 23rd National Conference of the ACM, pages 517-524. ACM Press, 1968.

11. Alain Trouvé. Diffeomorphisms groups and pattern matching in image analysis. International Journal of Computer Vision, 28(3):213-221, 1998.

12. X. Pennec and J.-P. Thirion. A framework for uncertainty and validation of 3D registration methods based on points and frames. IJCV, 25(3):203-229, 1997.

13. M. Tenenbaum and H. Pollard. Ordinary Differential Equations. Dover, 1985.

14. Michael Wüstner. A connected lie group equals the square of the exponential image. Journal of Lie Theory, 13:307-309, 2003. 
15. Luis Ibàñez, Will Schroeder, and the Insight Consortium. The ITK software Guide. The Insight Consortium, february 2003. http://www.itk.org/.

16. V. Arsigny, X. Pennec, and N. Ayache. A novel family of geometrical transformations: Polyrigid transformations. application to the registration of histological slices. Technical Report 4837, INRIA, May 2003.

17. E. Bardinet, S. Ourselin, G. Malandain, D. Tandé, K. Parain, N. Ayache, and J. Yelnik. Three dimensional functional cartography of the human basal ganglia by registration of optical and histological serial sections. In IEEE International Symposium on Biomedical Imaging, pages 329-332, Washington, USA, 2002. 